\title{
An Enhanced Queue Management Approach for Greedy Routing in MANETs
}

\author{
Ahmad Habboush $^{1} \&$ Mahmoud AlShugran ${ }^{1}$ \\ ${ }^{1}$ Faculaty of computer and information technology, Jerash Unuversity, Jordan \\ Correspondence: Ahmad Habboush, Faculaty of computer and information technology, Jerash Unuversity, Jerash, \\ jordan.E-mail: ahmad_ram2001@yahoo.com, shugran67@yahoo.com
}

\author{
Received: April 10, 2018 Accepted: April 17, $2018 \quad$ Online Published: April 25, 2018 \\ doi:10.5539/cis.v11n2p64 URL: http://dx.doi.org/10.5539/cis.v11n2p64
}

\begin{abstract}
Mobile Ad-hoc network (MANET) is the guiding technology of ubiquitous era and a cornerstone in the 4G communication architecture. MANET has limited resources and characterized with high mobility and frequent topology change. Routing in MANET should follow these constrains, otherwise, it can severely degrade the performance of MANET. Owing to Greedy routing (GFS) nature in MANET, a certain part of the MANET becomes congested before other parts. In such area the nodes have failed to forward packets in a timely manner or drop them, thus such nodes called Network Holes. This paper presents a simple and efficient enhanced GFS routing algorithm called Intelligent Fuzzy logic Greedy forwarding Scheme (IFGFS). IFGFS consider the De-congestion level of each mobile node as another selective metric besides distance. In order to validate our algorithm, we rely on a wide simulation experiments. The evaluation results showed the correctness of our new proposed algorithm the adaptive- congestion IFGFS which maximize the performance of the network.
\end{abstract}

Keywords: Mobile Ad-hoc network, Greedy routing, De-congestion level

\section{Introduction}

The functionality qualifications of Mobile Ad-hoc networks (MANETs) are envisioned to become an essential component of overall next-generation wireless network functionalities (Belkadi, 2010; Boukerche et al., 2011; Ambhaikar\& Sharma, 2010; Kavitha\&Vanaja, 2015). MANETs are networks formed without a central administration. They consist of mobile nodes on the fly. The mobile nodes in ad hoc networks communicate wirelessly, and can serve as both hosts and routers at the same time (Zheng et al., 2011; Hiremath\&Joshi, 2012; De Rango et al., 2012; Chen\&Weng, 2012; Rajaram\&Sugesh, 2011).

Due to its implementation simplicity and efficiency, Cartesian forwarding policy (Sandeep et al., 2017; Palaniappan, 2015; Mouzehkesh et al., 2013; Jannu\&Jana, 2016; Hassan, et al., 2014) is the most used with the majority of recently proposed position-based routing protocols. GFS follows the shortest path (optimal path), since it makes the largest possible movement towards the destination.

However, owing to adopting distance as the only deciding routing metric, the GFS is not always achieving the routing success because of many issues. This failure specifically appears in large-scale transmission of heavy traffic in MANET (Nasehi et al., 2013; Sangwan et al., 2013; Thayananthan\&Alzranhi, 2014; Smail et al., 2014; Sharma et al., 2013). In such environment, data packets loss and delay is a very familiar phenomenon due to congestion which appears as a result of the high number bits that holds error compared to the total sent number of bits in the wireless channel (Varaprasad, 2012; Hughes et al., 2012; Mamaghanian et al., 2011). The current design of traditional GFS is not congestion-adaptive, which consider as one of the main reasons behind its failure. Congestion problem required retransmission and rerouting lost packets again, which serve as another drawback in current GFS.

To solve the addressed problem, the congestion issue should be considered during the case of establishing an optimal multi-hop path between communicating nodes in MANET. To accomplish this goal, it is an important to select a node which can support reliable communication for an assigned number of times as the next-relay node. Reliability will be improved by adopting congestion factor besides distance to make the selection decision. This paper, introduces an Intelligent Fuzzy logic Greedy forwarding Scheme IFGFS routing protocol that complements the congestion problems that can occur in creating the multi-hop shortest route in MANET. IFGFS 
considers not only distance metric but also the de-congestion level of intermediate nodes as a multi- metric to select the optimal next relay node.

The rest of this article is organized as following: Section $\Pi$ presents relevant scholarship. Section $\amalg$ presents our proposal IFGFS. Part IV presents Fuzzify Input and Output Parameters. Section V presents performance analysis of the proposed IFGFS, followed by Conclusions in Section VI.

\subsection{Describe Relevant Scholarship}

Congestion in MANET incurs packet loss, retransmission and rerouting of lost packets which leads to more delays, and causes more energy consumptions. In the state of art several studies have been proposed which are tried to solve the congestion issue in the context of MANET.

In (Gulati\&Krishan, 2015) M.K. Gulati and Krishan Kumar presented a new protocol to provide an effective traffic balancing at each node so that a stable route could be guaranteed between two end systems. To enhance the network performance, their scheme employed several metrics such as delay, queue length and signal strength. The authors applied several experiment in order to investigate their scheme's correctness. The simulation results of the proposed protocol showed a high performance of the network. In (Jung, 2014) A. Jung proposed load balanced congestion adaptive routing (LBCAR). The main idea of LBCAR is to move traffic load from high congested nodes to de-congested nodes, in such way the performance of the network can be improved. The author used link cost and traffic load density to find the congestion status. The author showed that the performance of LBCAR is very high if it compared with other protocols.

In (Wei et al., 2016) Y., Wei et al. presented a queue management approach, their approach take into account the ability and the importance of the node in the network to make the queue threshold. Based on node's congestion situation it controls its buffer queue. The experiment results prove that the urged algorithm have the ability to save the weak nodes, and also can alleviate the congestion level of the network. In (Huang et al., 2013) H. Huang et al. presented an adaptive queue management algorithm API (Adaptive Proportional Integral). The proposed API provides the queue length through monitoring the router buffer queue size. The proposed API has the ability to adjust its parameters based on the current queue length. The authors used network simulator 2 in order to show the effectiveness of their algorithm. They improve that by using API in high dynamic network, the network has better robustness and stability, and low congestion.

In (Mallapur et al., 2015) S. V. Mallapur et al. proposed load balancing technique for congestion control (MLBCC) in Mobile Ad-hoc Networks. The main goal of MLBCC is to distribute the traffic load evenly for all routes in the network by minimizing congestion level. The MLBCC protocol makes two main functions firstly, the algorithm detect the congestion through using the arriving and an outgoing rate of traffic at a specific time. Secondly, the selection of suitable outgoing interface is totally based on the first criterion. The experiments results of the simulation showed high network performance when using MLBCC algorithm.

In (Deshmukh et al., 2014) S. R. Deshmukh and V. T. Raisinghani presented a new routing protocol concern about load balancing and energy efficiency. The new protocol hired multiple paths at the same time; it first discover neighbour then discover multipath and then transmits data. To investigate EALBM protocol many experiments have been done by using in Network Simulator 2. The experiments results showed that EALBM algorithm has better performs than other protocols. In (Xu et al., 2012) Y. Xu et al. presented a new routing metrics which are local data transmission rates, interference and packet drop rates. To validate the new routing metrics, the authors used simulation experiments. The simulation results showed that the new routing metric guarantee high load balancing in different aspects.

In (Othmen et al., 2016) S. Othmen et al. proposed a new scheme that can select a multi-path that satisfy the traffic load, energy conditions, and the requirements of QoS based on several criteria. The goal of the propose algorithm is to maximize the network lifetime. To validate the proposed algorithm, a vast number of experimental simulations have been performed by using Network Simulator 2. The results showed that the new algorithm is better than other current protocols regarding of Packet delivery ratio, end-to-end delay, and Network lifetime. In (Kumar et al., 2014) G. R. S., Kumar proposed a new cache queuing scheme. To validate the proposed algorithm, a vast number of experimental simulations have been performed by using Network Simulator 2. The results showed that the new algorithm show higher performance compared to other current protocols regarding the packet delivery ratio and achieves high throughput. In (Cheng et al., 2012), H., Cheng, et al. presented a new scheme to solve the problem of dynamic load balanced. To author proposed the vast types of dynamic genetic algorithms to solve this problem in MANETs.

The works introduced above tried to evaluate the level of activity in intermediate nodes by measuring either the 
load or the delay. What we can notice here that the current designs of routing protocol are not congestion-adaptive. Moreover, in the previous works routing may let a congestion happen and the goal behind optimal path establishment is trying to avoid the already become congested nodes. As an outset, overloading in this respondent mode increases latency and unnecessary packet loss and requires a significant increase in load if a new path is required.

\section{Method}

In Fuzzy logic Greedy forwarding Scheme (IFGFS) as a fuzzy logic controller (FLC) approach to become acclimatized to the greedy forwarding selection strategy in position based routing protocols, De-congestion Ratio $\left(\varphi_{n}\right)$ and Distance to Destination Ratio ${ }^{\left(\operatorname{des}_{n}^{a v a}\right)}$ are utilized as input parameter and to represent the output parameter we use the Nod's Reliability Ratio $\left(R_{n}\right)$. Thus, fuzzy logic controller for IFGFS approach chooses the most reliable neighbour depend on these two metric. Neighbours closest to destination and have high de-congestion level have highest reliability value. Similarly, neighbours farthest from destination and have low de-congestion level have lowest reliability value. Figure 1 shows the FLC for IFGFS approach.

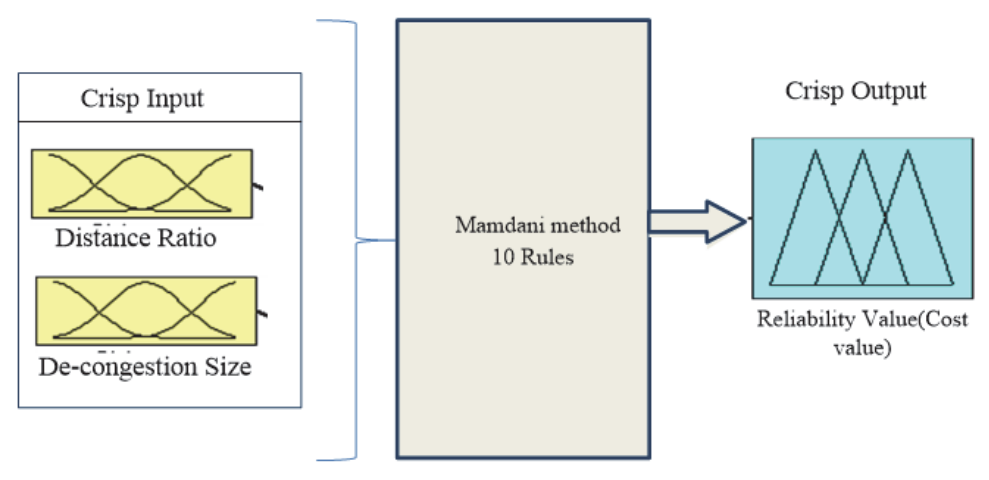

Figure 1. FLC for IFGFS

The enhanced forwarding scheme IFGFS constructed from two parts components. The first part presents the two metrics that will be used by the FLC to find the optimal neighbour (most reliable one). These metrics are the distance metric (traditional used metric), and the de-congestion level as new metric. The second part of the proposed work is the Inelegant Fuzzy Logic Routing Decision.

In this work, we aim to evolve a greedy-congestion aware forwarding protocol; our intended protocol will use the following routing metrics:

- $\quad$ Neighbours' De-Congestion Level

- Neighbours' Distance to Destination Ratio

With preference given to less congested and closest to destination neighbour as next relay hop to improve greedy performance.

\section{A. Neighbours De-Congestion Level Identification)}

To offer greedy routing more efficiency it is preferable to forward data packets via the least-congested neighbours in order to decrease latency resulted by queuing time. A node said to be congested if arrived number of data packets exceeds its buffer size. In such case, the node starts drop beckets which considered as lost packets. To monitor a node's congestion level there are a diverse of metrics that can be used. For the sake of IFGFS, this work inspired the metric used in (Tran et al., 2005). A node's Buffer Occupancy Ratio $\left(B^{r}\right)$ can be determined as a function varies with time. $B^{r}$ can be designated depending on the ratio between the numbers of data packets presently buffered to the buffer size of the nod. Regarding to $B^{r}$ values, a node's de-congestion statues $\left(\varphi_{n}\right)$ can be calculated. Both can be estimated using the following Equations in (1) and (2) respectively.

$$
B^{r}\left(t_{s}\right)=\frac{\text { occupied queue size }\left(t_{s}\right)}{\text { buffer size }}
$$

Each node uses IFGFS in MANET periodically checks the occupancy of its buffer. As a node with traditional GFS periodically reports its location and ID, in IFGFS each node also periodically transmit same beacon to its neighbours within its transmission radii. Figure 2 , shows the new structure of the beacon message $\left(b_{n}\right)$ used with 
IFGFS scheme. Every node in MANET estimates its buffer occupancy ratio firstly, and then it calculates its de-congestion level using Equation (2) below, and finally it appendstomessage. We can argue that the node is fully congested if the ratio is (zero) 0 , and far from congested if the ratio is (one) 1 . Thus, high values of neighbour's give an indicator for the high reliability value of communication via this neighbour.

$$
\varphi_{n}\left(t_{s}\right)=1-B^{r}\left(t_{s}\right)
$$

As showed in Figure 2, the initial beacon message hold 4 fields which are; node address ID, the geographical position of the node as $(x, y)$ coordinates, de-congestion ratio $\left(\varphi_{n}\right)$ at the node interface at time $\left(t_{s}\right)$, and the time that the neighbour transmitted $b_{n}$ message $t_{s}$.

\begin{tabular}{|c|c|c|c|c|}
\hline Node ID & \multicolumn{2}{|c|}{ Position Information } & $\begin{array}{c}\text { De-congestion } \\
\text { level }\end{array}$ & Sending Time \\
\hline \multirow{2}{*}{$I D_{t s}$} & $x_{c o}$ & $y_{c o}$ & $\varphi_{n}^{r}$ & $t_{s}$ \\
\cline { 2 - 3 } & $x_{t s}$ & $y_{t s}$ & & \\
\hline
\end{tabular}

Figure 2. Periodic beacon structure used in IFGFS

A sourcelforwarder node receives the new beacon message will renew and update its neighbours' matrix with the latest information reported in $b_{n}$, this matrix called Initial Parameters Value matrix (IPV). In case it receive this message for the first time from new neighbour it will imitate new entry for that neighbour, otherwise it will update the information regarded to the existed one. Figure 3, shows the structure of the node's neighbours' matrix IPV.

$$
\operatorname{IPV}=\left[\begin{array}{ccccc}
x_{1} & y_{1} & \varphi_{1}^{r} & t_{s 1} & I D_{1} \\
x_{2} & y_{2} & \varphi_{2}^{r} & t_{s 2} & I D_{2} \\
\cdot & \cdot & \cdot & \cdot & \cdot \\
\cdot & \cdot & \cdot & \cdot & \cdot \\
\cdot & \cdot & \cdot & \cdot & \cdot \\
x_{i} & y_{i} & v_{i}^{r} & t_{s i} & I D_{i}
\end{array}\right]
$$

Figure 3. IPV Structure with initial entries from $b_{n}$ beacon messages for $i$ neigbours

\section{B. Neighbours'Average Distance to Destination Identification}

A sourcelforwarder node that has (or received) data packet to be forwarded to a specific destination that located outside its transmission range behaves as follows. Firstly, it estimate the distance between itself and ultimate destination for later use. Next, it searches in its neighbours' PIV matrix looking for positive neighbours in the direction of ultimate destination. If it has not neighbour(s) satisfy its demand then it buffer the packets and continue moving until it finds its demand. Otherwise, and depending on the information related to each positive neighbour in the PIV matrix, source node start calculating the distance between each positive neighbour and the ultimate destination as shown in Equation (4) below.

$$
\operatorname{des}_{d}^{n}\left(\left(x_{t_{s}}^{n}, y_{t_{s}}^{n}\right)\left(x_{t_{s}}^{d}, y_{t_{s}}^{d}\right)\right)=\sqrt{\left(x_{t_{s}}^{n}-x_{t_{s}}^{d}\right)^{2}+\left(y_{t_{s}}^{n}-y_{t_{s}}^{d}\right)^{2}}
$$

Next, and to finalized neighbours' distance to destination, source node estimate the ratio of those neighbours' distances regarding to its own distance to destination as shown in Equation (4) and (5) below.

$$
\begin{gathered}
\operatorname{des}_{S}^{n}=\frac{\text { Positive neighbour distance to destination }}{\text { distance of source node S to the ultimate destination }} \\
\operatorname{des}_{n}^{\text {ava }}=1-\operatorname{des}_{S}^{n}
\end{gathered}
$$

We can say that the node is the closest to destination if the ratio is (one) 1 , and the farthest from destination if the 
ratio is (zero) 0 . Thus, high values of neighbour's give an indicator for the high reliability value of communication via this neighbour.

Finally, and depending on the new results in Equation (5), and the values of $\varphi_{n}$ : for each positive neighbour in IPV matrix, the sourcelforwarder node creates new matrix called Parameters Value matrix (PVM). The entries of this matrix consist of the positive neighbour ID, de-congestion level $\varphi_{n}$, and distance averagedes ${ }_{n}^{a v a}$. In this matrix the arrangement of positive neighbours will be in a descending order, the closest to destination will be in the first upper raw and with its relevant de-congestion value and identity number.

$$
\mathrm{PVM}=\left[\begin{array}{ccc}
\operatorname{des}_{1}^{\text {ava }} & \varphi_{1}^{r} & I D_{1} \\
\cdot & \cdot & \cdot \\
\cdot & \cdot & \cdot \\
\cdot & \cdot & \cdot \\
\operatorname{des}_{n}^{\text {ava }} & \varphi_{n}^{r} & I_{n}
\end{array}\right]
$$

Figure 4. PVM Structure with estimated entries for i neigbours

\section{Inelegant Fuzzy Logic Routing Decision}

The sourcelforwarder node can estimate the reliability level for each neighbour (depending on PVM matrix) by using IFGFS. Each entry in PVM matrix represents the estimated value for each neighbour. To estimate the reliability index for a neighbour, the corresponding entry will be evaluated by the Inelegant Fuzzy Logic Controller IFGFS. The crisp input will be the neighbour distance to destination and its de-congestion level. The crisp output from fuzzy controller will be the reliability index for that neighbour. The crisp output will shape a new entry in a new matrix called Reliability Estimation Values (REV). Figure 5, shows the content of REV. And finally, and depending on REV matrix, sourcelforwarder node can select the optimal neighbour as next relay node which has the highest reliability value in terms of distance and de-congestion level.

$$
\mathrm{REV}=\left[\begin{array}{cc}
I D_{g} & R_{g} \\
\cdot & \cdot \\
\dot{I} D_{n} & R_{n}
\end{array}\right]
$$

Figure 5. REV structure with reliability index estimated by IFGFS for $i$ neigbours

As it is detailed in Figure 5, REV contains the ID number of the positive neighbour nodes (it takes a random arrangements number $(\mathrm{g})$ according to reliability value from $(\mathrm{g}-n), n$ is the number of positive neighbours), and the reliability value for each one is $\mathrm{R}$.

Now, after firstly we get the fuzzy groups and then specify the degree of membership for every fuzzy group we then deploy the fuzzifier that will map the crisp data values. In our algorithm, the crisp input are $\operatorname{des}_{n}^{a v a}$ and $\varphi n$ hence, the Rn which is the crisp output will be fuzzified into five fuzzy groups as suggested by (Yan et al., 1994; Oussalah, 2001; Chandramohan et al., 2006), adjacent groups overlapped by $10 \%$ to $50 \%$.

\section{Results and Discussions}

In order to draws the crisp data values to fuzzy groups, the fuzzifier is used, thus we can assigns the degree of membership for each fuzzy set. In our approach the crisp input are $d e s_{n}^{a v a}$ and $\varphi_{\mathrm{n}}$ and thecrisp output is $\mathrm{R}_{\mathrm{n}}$ which we fuzzified to 5 fuzzy groups as suggested by (Yan et al., 1994; Oussalah, 2001; Chandramohan et al., 2006), adjacent groups overlapped by $10 \%$ to $50 \%$. In this paper we use the 0 to 1 range to normalize outputs and inputs linguistic values.

\section{A. Fuzzify Neighbours'Average Distance Input}

Membership functions can have different shapes. To show the entire set of medium values, we use the triangular membership function. The Z-shaped is used to show the entire group of low values. Also we use the S-shaped to show the entire group of high values. For the fuzzy groups for the $d e s_{n}^{a v a}$ input variable we hire the names as following: very close $(v c)$, close $(c)$, medium $(m)$, far $(f)$, and very far ( $v f)$. Table I shows the input des ava variable, which is has maximum value one, and minimum value zero. 
Table 1. Fuzzy groups of $\operatorname{des}_{n}^{a v a}$ input variable

\begin{tabular}{l|l}
\hline \multicolumn{1}{c|}{$\boldsymbol{d e s}_{\boldsymbol{n}}^{\text {ava }}$ ratio } & \multicolumn{1}{c}{ Fuzzy sets } \\
\hline $0.000-0.270$ & Very far $(v f)$ \\
$0.155-0.465$ & Far $(f)$ \\
$0.340-0.660$ & Medium $(m)$ \\
$0.535-0.845$ & Close $(c)$ \\
$0.720-1,000$ & Very close $(v c)$ \\
\hline
\end{tabular}

\section{B. Fuzzy Rules and Fuzzy Inference}

Fuzzy inference uses the following suggested fuzzy rules to map the fuzzy $\operatorname{des}_{n}^{\text {ava }}$ input groups mentioned above into fuzzy $R_{n}$ output groups: high reliable, reliable, medium, low reliable, and unreliable.

RULE 1: IF des $_{n}^{\text {ava }}$ is Very far THEN $R_{n}$ is unreliable

RULE 2: IF $\operatorname{des}_{n}^{\text {ava }}$ is far THEN $R_{n}$ is low reliable

RULE 3: IF des ava $_{n}^{\text {av }}$ is medium THEN $R_{n}$ is medium

RULE 4: IF des $_{n}^{\text {ava }}$ is close THEN $\mathrm{R}_{\mathrm{n}}$ is reliable

RULE 5: IF des ava is very close THEN $R_{n}$ is high reliable

\section{Fuzzify Neighbours' De-Congestion level}

Membership functions can have different shapes. We use the triangular membership function to show the entire group of medium values. The Z-shaped is used to model the entire group of low values, also we hire the S-shaped to show the entire group of high values. The fuzzy groups for the $\varphi \_n$ input variable have the following names: de-congested $(d c)$, semi-empty (se), fair $(f)$, congested $(c)$, and fully congested $(f c)$. Table $\Pi$ shows the assignment $\varphi$ variable, which is has maximum value one, and minimum value zero.

Table 2. Fuzzy groups of $\varphi$ input variable

\begin{tabular}{r|l}
\hline $\boldsymbol{\varphi}_{\boldsymbol{n}}$ ratio & Fuzzy sets \\
\hline $0.000-0.270$ & Fully congested $(f c)$ \\
$0.155-0.465$ & Congested $(c)$ \\
$0.340-0.660$ & Fair $(f)$ \\
$0.535-0.845$ & Semi-empty $(c e)$ \\
$0.720-1,000$ & De-congested $(d c)$ \\
\hline
\end{tabular}

D. Fuzzy Rules and Fuzzy Inference

Fuzzy inference uses the following suggested rules to map the fuzzy $\varphi$ input groups mentioned above into fuzzy R output groups: $\varphi_{\mathrm{n}}$.

RULE 6: IF $\varphi_{\mathrm{n}}$ is fully congested THEN $\mathrm{R}_{\mathrm{n}}$ is unreliable

RULE 7: IF $\varphi_{\mathrm{n}}$ is congested THEN $\mathrm{R}_{\mathrm{n}}$ is lowreliable

RULE 8: IF $\varphi_{\mathrm{n}}$ is fair THEN $\mathrm{R}$ is medium

RULE 9: IF $\varphi_{\mathrm{n}}$ is semiempty THEN $\mathrm{R}_{\mathrm{n}}$ is reliable

RULE 10: IF $\varphi_{\mathrm{n}}$ is verydecongested THEN $\mathrm{R}_{\mathrm{n}}$ is highreliable

\section{E. Fuzzify Neighbours' Reliability value Output}

Membership functions can have different shapes. We use the triangular membership function to show the entire group of medium, low, and high values. Fuzzy groups for the output variable $\mathrm{R}_{\mathrm{n}}$ take the next names: highreliable ( $h r)$, reliable $(r)$, medium $(m)$,lowreliable (lr), and unreliable (ur). Table W shows the membership functions and the assigned values of $R_{n}$ variable, which is has maximum value one, and minimum value zero. 
Table 3. Fuzzy groups of R output variable

\begin{tabular}{c|l}
\hline \multicolumn{1}{c|}{$\boldsymbol{R}_{\boldsymbol{n}}$ ratio } & Fuzzy sets \\
\hline $0.0000-0.3000$ & High reliable $(\mathrm{hr})$ \\
$0.1525-0.4725$ & Reliable $(r)$ \\
$0.3250-0.6750$ & Medium $(m)$ \\
$0.5250-0.8550$ & Low reliable $(l r)$ \\
$0.7000-1.0000$ & Un-reliable $(v l)$ \\
\hline
\end{tabular}

\section{F. The rule- base for IFGFS}

To implement IFGFS, the ten previous rules (R1 to R10) can be combined together to control the node reliability value $\mathrm{R}_{\mathrm{n}}$ based on $\varphi_{\mathrm{n}}$ and $\operatorname{des}_{n}^{a v a}$. The combination shaped one 2-dimensional rule-base as presented in Table IV.

Table 4. Rule-Base for IFGFS

\begin{tabular}{|l|l|l|l|l|l|}
\hline \multicolumn{1}{|c|}{$\begin{array}{l}\text { des ava }_{n} \rightarrow \\
\varphi_{n}\end{array}$} & very far & \multicolumn{1}{c|}{ far } & \multicolumn{1}{c|}{ medium } & \multicolumn{1}{c|}{ close } & \multicolumn{1}{c|}{ very close } \\
\hline fully congested & unreliable & unreliable & low reliable & low reliable & low reliable \\
\hline congested & unreliable & unreliable & low reliable & medium & low reliable \\
\hline fair & unreliable & low reliable & medium & medium & reliable \\
\hline semi-empty & medium & low reliable & medium & reliable & high reliable \\
\hline de-congested & medium & medium & reliable & high reliable & high reliable \\
\hline
\end{tabular}

These rules are used to formulate the conditional statements in fuzzy logic like:

IF $d e s_{n}^{a v a}$ is very far AND $\varphi_{\mathrm{n}}$ is fully congested THEN $\mathrm{R}_{\mathrm{n}}$ must be unreliable

The input to an 'IF- AND - THEN' rules are the numerical values of $\operatorname{des}_{n}^{a v a}$ and $\varphi_{\mathrm{n}}$ and the output is an entire fuzzy group (in this case, $R_{n}$ ). Then this group is defuzzified, assigning one numerical value to the $R_{n}$.

\section{G. Defuzzification}

Defuzzification is the final step; it indicates to the way a crisp value is obtained from a fuzzy group as an exemplification value. In literature there a numerous types of defuzzifiers, in our approach we use the Centroid method [35] for defuzzification, as in (9).

$$
R_{n}=\frac{\sum_{j=1}^{w} x_{j} \cdot \mu\left(x_{j}\right)}{\sum_{j=1}^{w} \mu\left(x_{j}\right)}
$$

\section{Performance Evaluation Of The IFGFS}

\section{A. Simulation Environment}

Simulating the suggested IFGFS method was performed by using Ns2 V2.33. For this simulation sake, we use the default group tings for the (GPSR*) GFS (the version with perimeter mode switched off, denoted as GPSR*) routing protocol. Also, in our experiments we use the Boundless model as a mobility model. The fuzzy logic system has been coded using $\mathrm{C}++$. Centroid was chosen as the defuzzification method. The routing buffer at the network layer could store up to 50 data packets.

In our experiments, rectangular simulation network of $2500 \mathrm{mx} 2000 \mathrm{~m}$ with transmission range up to $250 \mathrm{~m}$ is used. Sources transfer data at a fixed data rate of 5 packets / sec. The data packet size that is set to the default values is 512 bytes, also we set the beacon packet to 64-byte. We also use the medium access control model 802.11 DCF RTS / CTS protocol. The hired bandwidth is 2 Mbps.

All simulation results have been averaged over 30 simulation runs. The simulation conducted in two different speeds $(5 \mathrm{~m} / \mathrm{s}$, and $20 \mathrm{~m} / \mathrm{s})$ with various numbers of communicated pairs of nodes. We set $5,7,9,11$, and 15 pairs of communicated pairs of nodes. The simulation is accomplished in 1200 seconds, and we just collect the results in the period of $800-1000$ second in the simulation lifetime in order to prevent the influence unreliable results.

\section{B. Performance Metrics}


To show the effectiveness and efficiency of the proposed solution we deploy two performance metrics. These metrics are packet loss rate and end to end dely.

\section{Simulation Results and Evaluations}

Figures 6 and 7, show the simulation results in MANET while we use our proposed protocol. The results show improvement increases with the mobility of the nodes. When the packet loss rate was around $12 \%$ for GPSR*, Intelligent Fuzzy logic Greedy forwarding Scheme decreased the packet loss rate by more than 55\%. This can be refer to the adaptive selection process in IFGFS, where GPSR* cannot select the next relay node in terms of congestion metric. Meanwhile, in the $20 \mathrm{~m} / \mathrm{s}$ and $5 \mathrm{~m} / \mathrm{s}$ cases, the average delay to transmit a packet went up slower using Intelligent Fuzzy logic Greedy forwarding Scheme when the traffic load increased.

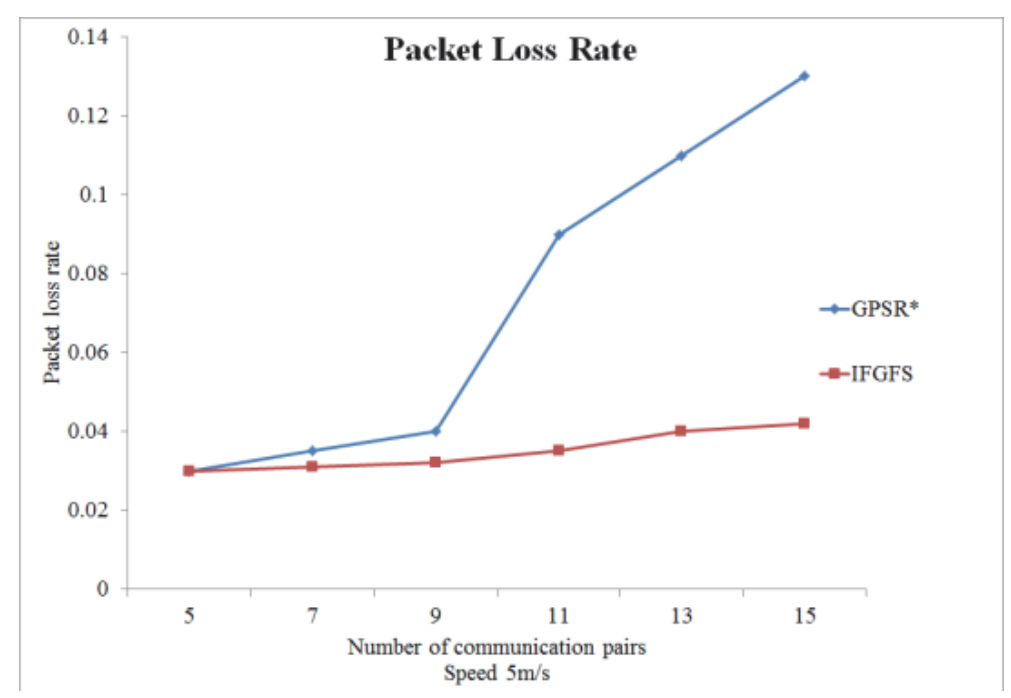

Figure 6(a).

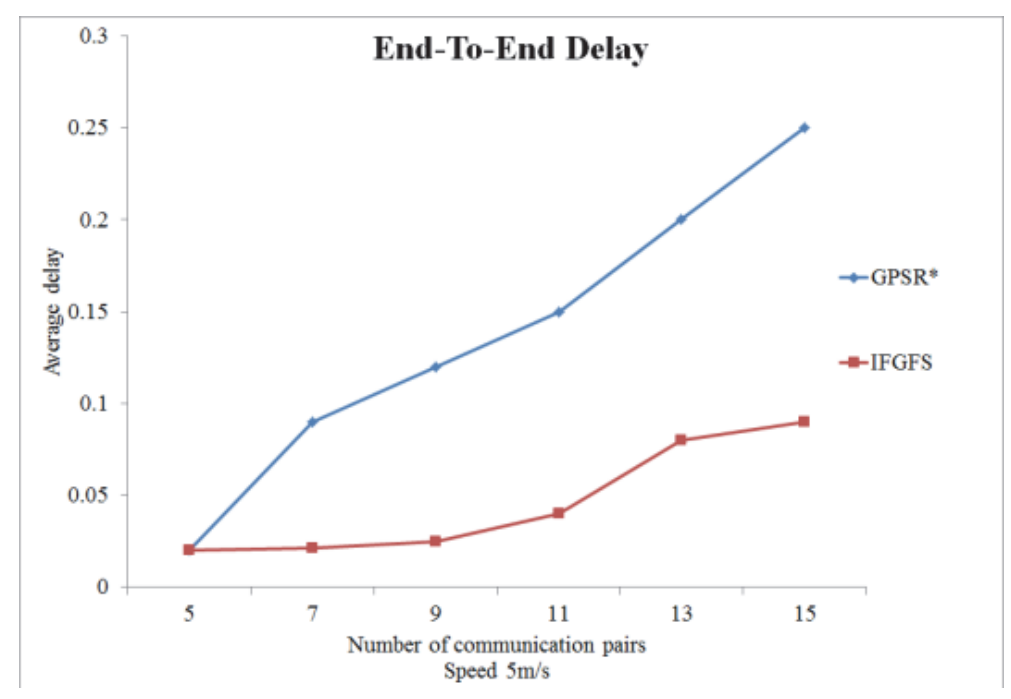

Figure 6(b).

Figure $6(\mathrm{a}, \mathrm{b})$. System Performance with Moving Speed of $5 \mathrm{~m} / \mathrm{s}$ 


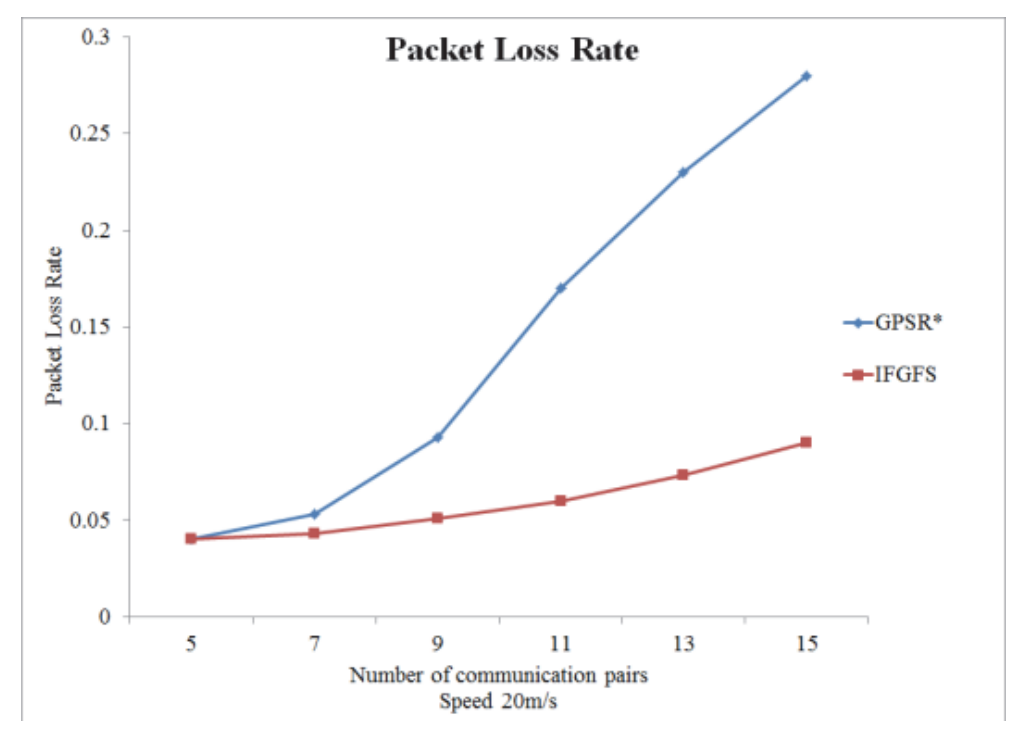

Figure 7(a).

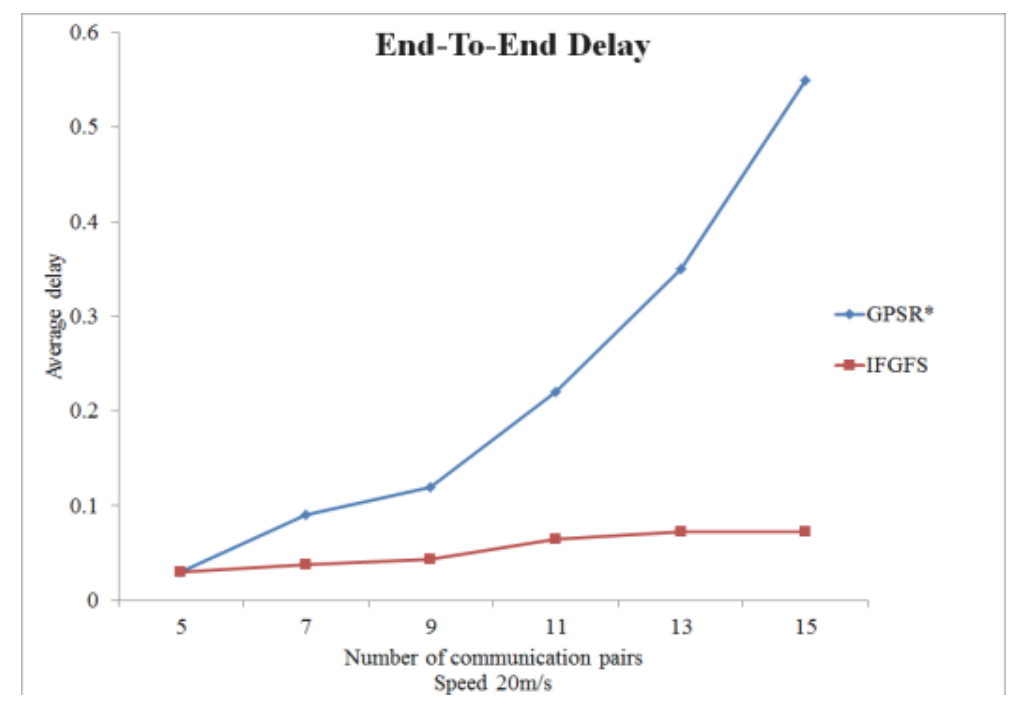

Figure 7(b).

Figure $7(a, b)$. System Performance with Moving Speed of $20 \mathrm{~m} / \mathrm{s}$

\section{Conclusions}

This work has shown how fuzzy logic can be successfully employed to make selection decision of the next relay node. The distance and de-congestion level for each neighbor are considered as crisp input. The defuzzified value of a fuzzy decision was used to weight the two input so that the best next relay node can be selected amongst other neighbours. It is improved that this would force avoidance of hot-spot areas in the center of a MANET though it may results to create a route that is longer than the shortest path generated by just employed hop count metric. On the other hand, and which is most important, such adaption improved the reliability level in terms of decreasing latency, and packet loss rate. As an outcome, the proposed protocol outperforms the traditional one which was experimentally improved.

\section{References}

Ambhaikar, A., \& Sharma, L. (2010). Exploring the Behavior of Mobile Ad Hoc Network Routing Protocols with Reference to Speed and Terrain Range," in the Proceeding of the International Multi Conference of Engineers and Computer Scientists (IMECS10), Hong Kong,798-803.

Belkadi, M. (2010). Intelligent Routing and Flow Control in MANETs.Journal of Computing and Information Technology - CIT, 3(2010), 233-243. 
Boukerche, A., Turgut, B., Aydin, N., Ahmad, M., Bölöni, L., \&Turgut, D. (2011). Routing protocols in ad hoc networks: A survey. Comput. Netw, 55, 3032-3080.

Chandramohan, A. et al. (2006). Two New and Useful Defuzzification Methods Based on Root Mean Square Value. Soft Computing-A Fusion of Foundations, Methodologies and Applications, 10,1047-1059.

Chen, C. W., \& Weng, C. C. (2012). A power efficiency routing and maintenance protocol in wireless multi-hop networks. J. Syst. Softw., 85(1),62-76.

Cheng, H., Yang, S., \& Cao, J. (September 2012). Dynamic Genetic Algorithms for the Dynamic Load Balanced Clustering Problem in Mobile Ad Hoc Networks.Expert Systems with Applications, 5.

De Rango, F., Guerriero, F., \& Fazio, P. (Apr. 2012). Link-stability and energy aware routing protocol in distributed wireless networks. IEEE Trans. Parallel Distrib. Syst., 23(4),713-726.

Deshmukh, S. R., \& Raisinghani, V. T. (2014). EALBM: Energy aware load balancing multipath routing protocol for MANETs," 2014 Eleventh International Conference on Wireless and Optical Communications Networks (WOCN), Vijayawada, 2014,1-7. https://doi.org/10.1109/WOCN.2014.6923061

Gulati, M.K., \&Krishan, K. (2015). Stable Energy efficient QoS based Congestion and Delay aware Routing (SEQCDR) Protocol for MANETs. IEEE ICCSP Conference,1-7.

Hassan, M. M., Kamruzzaman, S. M., Alamri, A., Almogren, A., Alelaiwi, A., Alnuem, M., Islam M. M., \& Razzaque, M. A. (2014). Design of an energy-efficient and reliable data delivery mechanism for mobile ad hoc networks: A cross-layer approach. Concurrency and Computation: Practice and Experience, 27, 2637-2655.

Hiremath, P., \& Joshi,S. M. (2012). Energy efficient routing protocol with adaptive fuzzy threshold energy for MANETs.Int. J. Comput. Netw. Wireless Commun., 2(2),402-407.

Huang, H., Xue, G., Wang, Y., \& Zhang, H. (2013). An adaptive active queue management algorithm," 2013 3rd International Conference on Consumer Electronics, Communications and Networks, Xianning, 72-75. https://doi.org/10.1109/CECNet.2013.6703275

Hughes, L., Wang, X., \& Chen, T. (2012). A Review of Protocol Implementations and Energy Efficient Cross-Layer Design for Wireless Body Area Networks. Sensors, 12, 14730-14773.

Jannu, S., \& Jana, P. K. (2016). Energy Efficient Algorithms for Hot Spot Problem in Wireless Sensor Networks. In Proceedings of the Second International Conference on Computer and Communication Technologies 2016, (pp. 509-517). Springer India.

Jung, A. (2014). Load Balanced Congestion Adaptive Routing for Mobile Ad Hoc Networks.International Journal of Distributed Sensor Networks, 2014(2014), Article ID 532043.

Kavitha, S., \& Vanaja, B. (2015). Survey on Energy Efficient Geographic Routing Protocols in Wireless Sensor Networks.International Journal of Innovative Research in Computer and Communication, 3(11), November 2015.

Kumar, G. R. S., \& Sundaram, K. M. M. (2014). Queuing based load balancing approach for improving network performance in manet. International Journal of Inventions in Computer Science and Engineering.

Mallapur, S. V., Patil, S. R., \& Agarkhed, J. V. (2015). Multipath Load Balancing Technique for Congestion Control in Mobile Ad Hoc Networks. 2015 Fifth International Conference on Advances in Computing and Communications (ICACC), Kochi, 2015,204-209.https://doi.org/10.1109/ICACC.2015.97.

Mamaghanian, H., Khaled, N., Atienza, D., \& Vandergheynst, P. (2011). Compressed Sensing for Real-Time Energy-Efficient ECG Compression on Wireless Body Sensor Nodes. IEEE Trans. Biomed. Eng., 58, 2456-2466.

Mouzehkesh, N., Zia, T., \&Shafigh, S. (April 2013). Traffic aware fuzzy-tuned delay range for wireless body area networks medium access control protocol (MAC). Proceedings of the 2013 IEEE Eighth International Conference on Intelligent Sensors, Sensor Networks and Information Processing, Melbourne, Australia, $2-5$.

Nasehi, H., Javan, N. T., Aghababa, A. B., \& Birgani, Y. G. (Mar. 2013). Improving energy efficiency in manets by multi-path routing.

Othmen, S., Zarai, F., Belghith, A., \& Kamoun, L. (2016). Energy, load, and QoS-aware routing protocol for Ad Hoc networks. 2016 International Wireless Communications and Mobile Computing Conference (IWCMC), 
Paphos, 2016,886-891. https://doi.org/10.1109/IWCMC.2016.7577175

Oussalah, M. et al. (2001). A New Derivation of Centroid Defuzzification,” 2001.

Palaniappan, C. (2015). Energy-efficient stable routing using QoS monitoring agents in MANET.Journal on Wireless Communications and Networking, 2015, 1-11.

Rajaram, D. A., \& Sugesh, J. (2011). Power aware routing for MANET using on demand multi path routing protocol. Int. J. Comput. Sci. Issues, 8,517-522.

Sandeep, S., Rajesh, M., \& Siddharth, D. (2017). Reliable Data Delivery Mechanism for Mobile Ad Hoc Network Using Cross-Layer Approach, Next- Generation Networks, 467-477. https://doi.org/10.1007/978-981-10-6005-2_47

Sangwan, R., Duhan, M., \& Dahiya, S. (2013). Energy Consumption analysis of ad hoc routing protocols for different energy models in MANET. IOSR Journal of Electronics and Communication Engineering, 6(4), $48-55$.

Sharma, D. K., Patra, A. N., \&Kumar, C. (2013). An update based energy-efficient reactive routing protocol for mobile ad hoc networks.Int. J. Comput. Netw. Inf. Secur. (IJCNIS), 5(11).

Smail, O., Cousin, B., Mekki, R., \& Mekkakia, Z. (2014). A multipath energy-conserving routing protocol for wireless ad hoc networks lifetime improvement. EURASIP J. Wireless Commun. Netw., 2014, 2014.

Thayananthan, V., \& Alzranhi, A. (2014). Enhancement of Energy Conservation Technologies in Wireless Sensor Network.Procedia Comput. Sci., 34,79-86.

Tran, D. A., \& Raghavandra, H. (Mar. 2005.). Routing with Congestion Awareness and Adaptively in Mobile Ad Hoc Networks." In Proceedings of wireless communication and networks conferences IEEE WCNC, Page(s): 1988 - 1994 Vol. 4.

Varaprasad, G. (2012). Stable routing algorithm for mobile ad hoc networks using mobile agent. International Journal of Communication System, 2012.

Wei, Y., Chang, L., Wang, Y., \& Wang, G. (2016). The congestion control algorithm based on queue management of each node in mobile ad hoc networks AIP Conference Proceedings, 1790, 150037.

Xu, Y., Deng, J. D., \& Nowostawski, M. (2012). Optimizing Routing in Multi-hop Wireless Networks Using Analytical Capacity Estimation: A Study on Video Streaming. 2012 IEEE 14th International Conference on High Performance Computing and Communication \& 2012 IEEE 9th International Conference on Embedded Software and Systems, Liverpool, 2012, pp. 748-755.doi: 10.1109/HPCC.2012.106

Yan, J., Ryan, M., \& Power, J. (1994). Using Fuzzy Logic: Towards Intelligent Systems. USA: Prentice Hall.

Zheng, S., Wu, W. Q., \& Zhang, Q. (2011). Energy and link-state based routing protocol for MANET", IEICE Trans. Inf. Syst., 94,1026-1034.

[1] (Belkadi,2010)

[2] (Boukerche et al., 2011)

[3] (Ambhaikar\& Sharma,2010)

[4] (Kavitha\&Vanaja,2015)

[5] (Zhenget al., 2011)

[6] (Hiremath\&Joshi,2012)

[7] (De Rangoet al., 2012)

[8] (Chen\&Weng,2012)

[9] (Rajaram\&Sugesh,2011)

[10] (Sandeepet al.,2017)

[11] (Palaniappan,2015)

[12] (Mouzehkeshet al., 2013)

[13] (Jannu\&Jana,2016)

[14] (Hassan, et al., 2014) 
[15] (Nasehiet al., 2013)

[16] (Sangwanet al., 2013)

[17] (Thayananthan\&Alzranhi,2014)

[18] (Smailet al., 2014)

[19] (Sharmaet al., 2013)

[20] (Varaprasad,2012)

[21] (Hugheset al., 2012)

[22] (Mamaghanianet al., 2011)

[23] (Gulati\&Krishan,2015)

[24] (Jung,2014)

[25] (Weiet al., 2016)

[26] (Huanget al., 2013)

[27] (Mallapuret al., 2015)

[28] (Deshmukhet al., 2014)

[29] (Xuet al., 2012)

[30] (Othmenet al., 2016)

[31] (Kumaret al., 2014)

[32] (Chenget al., 2012)

[33] (Tranet al., 2005)

[34] (Yanet al., 1994)

[35] (Oussalah,2001)

[36] (Chandramohanet al., 2006)

\section{Copyrights}

Copyright for this article is retained by the author(s), with first publication rights granted to the journal.

This is an open-access article distributed under the terms and conditions of the Creative Commons Attribution license (http://creativecommons.org/licenses/by/4.0/). 